\title{
DIABETES MELLITUS E OBESIDADE COMO FATORES DE RISCO PARA INCONTINÊNCIA URINÁRIA EM MULHERES NO PRÉ-OPERATÓRIO DE INCONTINÊNCIA URINÁRIA DE ESFORÇO
}

Danielle Caroline Manfre ${ }^{1}$, Débora Maria Gonçalves Santoro², Camila Pereira Silvério², Mariane Costa Christovam², Oscar Rubini Ávila ${ }^{3}$, Francis Lopes Pacagnelli ${ }^{2}$, Gabriela Andrade Piemonte Lopes $^{2}$

${ }^{1}$ Fisioterapeuta, Presidente Prudente - SP. ${ }^{2}$ Universidade do Oeste Paulista - UNOESTE. Curso de Fisioterapia, Presidente Prudente, SP. Curso de Medicina, Presidente Prudente, SP. e-mail: gabriela@uneste.br

\section{RESUMO}

Detectar a obesidade e o diabetes mellitus em mulheres no climatério ou menopausa com indicação o tratamento cirúrgico de IUE. Estudo realizado com 5 mulheres no Hospital Regional de Presidente Prudente-SP. Foram avaliados, o índice de massa corpórea, circunferência abdominal, características sócio demográficas, obstétricas, ginecológicas, morbidades, atividade física habitual, e qualidade de vida. A idade foi de $56,8 \pm 11,58$ anos, IMC de $24,3 \pm 5,11 \mathrm{~kg} / \mathrm{m}^{2}, 60 \%$ das pacientes com CA $>80 \mathrm{~cm}$, o KHQ obteve nos domínios de percepção saúde $50 \pm 17,67$, impacto incontinência 79,6 $\pm 18,62$, limitações das atividades diárias 53,2 $\pm 36,07$, limitações físicas $79,6 \pm 14,22$, limitações sociais $50,8 \pm 37,30$, relações pessoais $49,8 \pm 37,23$, emoções $77,6 \pm 34,39$, sono/ energia $66,4 \pm 35,59$ e medidas de gravidade $49,2 \pm 24,24$, e $40 \%$ das pacientes com diabetes tipo 2. A obesidade abdominal, idade e tipos de parto mostraram-se ser importantes fatores de risco para o desenvolvimento da IU, implicando na elaboração de intervenções nos vários níveis de atenção à saúde no tratamento da IU.

Palavras chave: Incontinência urinária, Obesidade, Diabete, Cirurgia

\section{OBESE AND DIABETES MELLITUS AS RISK FACTORS FOR URINARY INCONTINENCE IN WOMEN IN PRE SURGICAL STRESS URINARY INCONTINENCE}

\begin{abstract}
To detect obesity and diabetes mellitus in women during the climacteric or menopause that were requested surgical treatment of SUI. A study conducted with 5 women in Hospital Regional of Presidente Prudente-SP. Were evaluated Body Mass Index, abdominal circumference, socio demographic, obstetric and ginecological characteristics, history of morbidity, the physical activity, and quality of life. The mean age of was $56.8 \pm 11.58$ years, BMI $24.3 \pm 5.11 \mathrm{~kg} / \mathrm{m}^{2}, 60 \%$ of patients with $W C>80 \mathrm{~cm}, \mathrm{KHQ}$ has obtained data in the fields of perception health $50 \pm 17.67$, incontinence Impact $79.6 \pm 18.62$, limitations of daily activities $53.2 \pm 36.07,79.6 \pm 14.22$ physical limitations, social limitations $50.8 \pm 37.30$, personal relations $49.8 \pm 37.23,77.6 \pm 34.39$ emotions, sleep/energy $66.4 \pm 35.59$, and severity measures $49.2 \pm 24.24$, and $40 \%$ of patients with diabetes mellitus type 2 . Abdominal obesity, age and mode of delivery were found to be important risk factors for the development of $\mathrm{UI}$. Our findings might have important implications for the development of interventions at various levels of health care, from prevention to treatment of UI .
\end{abstract}

Keywords : Urinary incontinence, Obesity, Diabetes, Surgery. 


\section{INTRODUÇÃO}

O Diabetes Mellitus (DM) está entre umas das principais doenças crônicas com maior número de morbimortalidade no Brasil. O número estimado de pessoas com DM é de aproximadamente cinco milhões, sendo que em 2025 calcula-se que este número alcance onze milhões no país ${ }^{1 ; 2}$, sendo atribuído à alguns fatores que predispõe o indivíduo a desenvolver o DM como o envelhecimento da população, sedentarismo, obesidade, estilo de vida, e mudanças nos padrões dietéticos ${ }^{3 ;} 4$, seus fatores podem ser hereditários e ambientais, trata-se de uma doença metabólica, que se apresenta pelo aumento da glicemia no sangue.

A obesidade é definida pela Organização Mundial da Saúde (OMS), como o acúmulo anormal ou excessivo de gordura, sendo estimada pelo Índice de Massa Corpórea (IMC), o qual é calculado dividindose $o$ peso (em quilogramas) pela altura (em metros) ao quadrado 5 .

A obesidade e o DM estão entre os fatores de risco para o desenvolvimento da Incontinência Urinária (IU), pois o aumento do peso corpóreo (principalmente da gordura abdominal) aumenta a pressão intraabdominal e a pressão intra-vesical, ocasionando fadiga dos Músculos do Assoalho Pélvico (MAP), alterando, assim, o bom funcionamento do trato urinário ${ }^{6}$. O DM se encaixa nos fatores dentro das doenças crônicas, onde a hiperglicemia causa um aumento na frequência urinária, alteração na força e na função mecânica dos MAP, sendo que aproximadamente $52 \%$ das pacientes hiperglicêmicas apresentam distúrbios nesse sistema ${ }^{7}$.

A IU, foi definida atualmente pela International Continence Society (ICS) como queixa de qualquer perda involuntária de urina, que constitui um problema social de higiene $^{6}$. É classifica em 3 tipos, Incontinência Urinária de Esforço (IUE), Incontinência Urinária de Urgência (IUU), e Incontinência Urinária Mista (IUM) ${ }^{8}$.

Segundo Guarisi et al., a incontinência urinária é um problema comum que pode afetar mulheres de todas as idades. Constitui de sintoma com implicações sociais, causa desconforto, perda de autoconfiança e interfere negativamente na qualidade de vida de muitas mulheres, além de representar um problema de saúde pública ${ }^{9}$.

Estudos mostram que a IU afeta diretamente a qualidade de vida dessas pessoas, sua autoestima, sua independência para a vida social e atividades do dia-a-dia, além dos problemas físicos. As pesquisas, também, apontam que esses pacientes sofrem com sentimento de vergonha e baixa autoestima perante a sociedade, apresentam alterações nas atividades sexuais e domésticas ${ }^{10}$. 
A IU de maneira geral, causa um grande impacto sócio econômico para o sistema de saúde pública no Brasil. A falta de informação dos profissionais de saúde ${ }^{11}$, especialmente, a respeito dos fatores de risco, dificulta a prevenção, o diagnóstico e o tratamento ${ }^{6}$.

O objetivo deste estudo baseou-se em identificar o diabetes mellitus, a obesidade, a atividade física e a qualidade de vida na incontinência urinária, em mulheres que no pré-operatório de incontinência urinária de esforço, e por meio dos resultados proporcionar meios para uma intervenção mais direcionada no que diz respeito à intervenção primária.

\section{MATERIAIS E MÉTODOS}

O estudo caracterizou-se por uma pesquisa observacional transversal que foi feito a partir da avaliação de mulheres submetidas ao tratamento cirúrgico de IUE de um hospital público em Presidente Prudente - SP, no período de dezembro de 2013 a fevereiro de 2014.

Primeiro foi lido o Termo de Consentimento Livre e Esclarecido, e após aceitarem participar da pesquisa assinaram o termo e posteriormente foi dado início ao questionamento sobre as variáveis de estudo.

A obesidade foi avaliada por meio do índice de massa corporal (IMC) e medida da circunferência abdominal (CA). O peso foi medido em quilograma por uma balança digital (Plena $\left.{ }^{\circledR}\right)$. A mensuração foi realizada duas vezes ${ }^{12}$.

A altura foi avaliada em metros por um estadiômetro em uma parede com $90^{\circ}$ em relação ao chão e sem rodapés. A leitura da medida foi realizada duas vezes ${ }^{13}$.

O IMC foi calculado a partir da divisão da massa corporal em quilogramas pela estatura em metros elevada ao quadrado $\left(\mathrm{Kg} / \mathrm{m}^{2}\right)$. De acordo com as Diretrizes Brasileiras de Obesidade $^{14,}$ o IMC é estratificado em: baixo peso IMC $<18,5$, peso normal 18,5-24,9, sobrepeso 25,0-29,9, obeso | 30,0-34,9, obeso II 35,0-39,9 e obeso III IMC $\geq 40$, classificação essa adaptada pela OMS (2000). Para o presente estudo, foram consideradas acima do peso todas as mulheres classificadas com IMC $\geq 25,0 \mathrm{~kg} / \mathrm{m}^{2}$.

A CA foi avaliada segundo Park et al, ${ }^{15}$ por meio da média de duas medidas realizadas com uma fita métrica, em milímetro e inelástica. Foi encontrado o ponto médio entre o ápice da crista ilíaca e a última costela do lado direito, e efetuada a medida do lado esquerdo no final de uma expiração, com o local desnudo. A medida da CA mostra o conteúdo de gordura visceral de acordo com as Diretrizes Brasileiras de Obesidade $^{14}$. A análise deste estudo considerou como obesidade abdominal uma CA acima de $80 \mathrm{~cm}$. 
As co-variáveis que favorecem o aparecimento da incontinência urinária foram investigadas por meio do questionamento da idade ${ }^{16}$, história obstétrica $^{17 ;}$, história de morbidade ${ }^{12}$, atividade física habitual International Physical Activity Questionnaires (IPAQ) ${ }^{18,}$ e 0 questionário de qualidade de vida "King's Health Questionnaire - KHQ's"19. Para a análise dos resultados foi utilizada estatística descritiva.

O estudo foi aprovado pelo Comitê de Ética e Pesquisa da Universidade do Oeste Paulista - UNOESTE, parecer no 1800 , e está de acordo com a Resolução do CONEP 466/2012.

\section{RESULTADOS}

Para a avaliação do presente estudo, foram selecionadas e convocadas 11 mulheres, onde dessas, apenas 5 compareceram no local e data agendada, não tendo justificativa do não comparecimento das demais.

A idade, IMC, CA e as características obstétricas das pacientes no pré-operatório de IUE são expostas na Tabela 1.

Tabela 1. Idade, IMC, CA e as características obstétricas das pacientes no pré-operatório de IUE

\begin{tabular}{llcc}
\hline \multicolumn{1}{c}{ Parâmetros } & & & \\
\hline Idade (anos) & & 56,8 & $\pm 11,58$ \\
IMC $\left(\mathrm{km} / \mathrm{m}^{2}\right)$ & & 24,3 & $\pm 5,11$ \\
$\mathrm{CA}(\mathrm{cm})$ & $\leq 80$ & $\mathrm{~N}$ & $\%$ \\
& $>80$ & 2 & 40 \\
Gestações & Nulíparas & 3 & 60 \\
& $1-3$ & 0 & 0 \\
& $\geq 4$ & 3 & 60 \\
Partos Vaginais & 0 & 2 & 40 \\
& $1-2$ & & 20 \\
Partos Cesáreas & $\geq 3$ & 1 & 40 \\
& 0 & 2 & 40 \\
& $1-2$ & & 60 \\
Abortos & $\geq 3$ & 3 & 20 \\
& & 1 & 20 \\
& sim & 1 & 0 \\
\hline
\end{tabular}

IMC - Índice de Massa Corpórea; CA - Circunferência Abdominal 
Das mulheres avaliadas $80 \%$ estavam no período do climatério/menopausa, e quanto as morbidades, $40 \%$ apresentavam diabetes mellitus e hipertensão arterial, e $20 \%$ eram cardiopatas.

Tabela 2. Tabela da avaliação dos escores da qualidade de vida, segundo os domínios do King's Health Questionnnaire (KHQ)

\begin{tabular}{lc}
\hline Domínios do KHQ & Média \pm Desvio Padrão \\
\hline Percepção da Saúde & $50 \pm 17,67$ \\
Impacto da Incontinência & $79,6 \pm 18,62$ \\
Limitacões das Atividades & \\
Diárias & $53,2 \pm 36,07$ \\
Limitações Fisícas & $79,6 \pm 14,22$ \\
Limitacões Sociais & $50,8 \pm 37,30$ \\
Relações Pessoais & $49,8 \pm 37,23$ \\
Emoções & $77,6 \pm 34,39$ \\
Sono/Energia & $66,4 \pm 35,59$ \\
Medidas de Gravidade & $49,2 \pm 24,24$ \\
\hline
\end{tabular}

Os resultados do nível de atividade física das mulheres avaliadas, realizado por meio do questionário IPAQ apresentaram $60 \%$ insuficientemente ativas, $20 \%$ ativa e $20 \%$ muito ativas.

\section{DISCUSSÃO}

Observou neste estudo as características que contribuíram para o desenvolvimento da IU, além do impacto desta disfunção na qualidade de vida da mulher incontinente.

Dados de um estudo que avaliou 80 mulheres com IU, mostrou uma média etária de 52,17 anos, o que corrobora com este
Observa-se na Tabela 3 que, os escores dos domínios do questionário de qualidade de vida KHQ's. 
indicando que a gordura visceral pode ser um fator importante para na disfunção do trato urinário, por aumentar o peso e ter a necessidade de uma maior força dos MAP para sustentação ${ }^{21}$.

Nosso estudo está de acordo com um estudo realizado na Korea, com 769 mulheres, que apesar do IMC ter média de $24,6 \mathrm{~kg} / \mathrm{m}^{2}$, a média da CA das mulheres avaliadas foi de $83,7 \mathrm{~cm}$, revelando que a obesidade abdominal é um importante fator de risco para o desenvolvimento da IUE ${ }^{22}$.

Estudos que enfatizam o DM como fator predisponente à IU devido ao maior volume urinário que a hiperglicemia causa, aumentando assim a freqüência urinária, respectivamente, vem sendo mais facilmente encontrados na literatura ${ }^{6 ; 23}$, justificando os $40 \%$ das pacientes estudadas em nosso estudo com diabetes mellitus previamente diagnosticadas.

Das avaliações realizadas em nossa pesquisa, nenhuma das mulheres eram nulíparas, o que segue de acordo com o estudo de Oliveira et al., que mostrou que o parto vaginal é o maior responsável por danos na musculatura dos MAP e à mecânica da $I^{24}$.

Com relação ao parto cesárea, Knorst et al apresentou $17,2 \%$ da amostra com esta via de parto, não estando de acordo com o presente estudo, que obteve $40 \%$ de mulheres com história de parto cesárea. No mesmo estudo, a episiotomia apresentou-se em $65,9 \%$ da amostra, e no estudo aqui descrito somente $20 \%$ exibiu a episiotomia na história obstétrica ${ }^{25}$.

Fatores mecânicos, como obesidade e tipo de parto, parecem estar mais associados com a presença da incontinência, e em geral, estes efeitos são independentes da idade da paciente ${ }^{28}$.

Leroy et al., apresentaram em recente pesquisa quando aplicado o King's Health Questionnaire - KHQ, que todos os domínios ou atingiram valores próximos a 50 ou ultrapassaram, como este estudo, que apresentou os domínios de Impacto da Incontinência, Limitações Físicas e Emoções, o que condiz com o estudo supracitado, que obteve o domínio Limitações das Atividades Diárias empatadas com Limitações Físicas ${ }^{27}$.

Virtuoso et al, em estudo que avaliaram a presença da incontinência urinária e função muscular perineal em idosas praticantes e não-praticantes de atividade física regular, concluíram que a prevalência de IU foi maior no grupo praticantes de atividade física, sugerindo influência da variável idade no mecanismo de continência urinária ${ }^{28}$. No presente estudo foi apresentado um maior número de mulheres insuficientemente ativas, com incontinência urinária, de acordo com o instrumento IPAQ utilizado neste, com um percentual de $60 \%$ 
das mulheres avaliadas, o que não condiz com este estudo.

Há uma proporção elevada de mulheres que relatam perda urinária durante suas atividades diárias e procuram tratamento, sendo estes a intervenção cirúrgica e tratamento fisioterapêutico. Apresentadas as características de mulheres com IU, a abordagem do tratamento fisioterapêutico vem sendo cada vez mais evidenciado, com a necessidade de maior conhecimento pela população incontinente, e melhor orientação pelos médicos. $\mathrm{O}$ tratamento conservador apresenta mínimos efeitos colaterais, e provoca menor constrangimento, melhorando a qualidade de vida dessas mulheres.

\section{CONCLUSÃO}

Conclui-se então, no presente estudo que, principalmente a obesidade abdominal, idade e tipos de parto mostraram-se ser importantes fatores de risco para 0 desenvolvimento da IU. Nossos achados devem ter implicações importantes para a elaboração de intervenções nos vários níveis de atenção à saúde, desde a prevenção até o tratamento da IU, refletindo na melhora da qualidade de vida destas pacientes.

\section{REFERÊNCIAS}

1. Castro ARV, Grossi SAA. Reutilização de seringas descartáveis no domicílio de crianças e adolescentes com diabetes mellitus. Rev Esc Enferm USP. 2007; 41 (2):187-95. http://dx.doi.org/10.1590/S0080$\underline{62342007000200003}$

2. Brasil. Ministério da Saúde. Coordenação Nacional do Plano de Reorganização da Atenção à Hipertensão Arterial e ao Diabetes Mellitus. Manual de hipertensão arterial e diabetes. Brasília; 2002.

3. Brasil. Ministério da Saúde. Avaliação do Plano de Reorganização da Atenção à Hipertensão Arterial e ao Diabetes Mellitus no Brasil. Brasília; 2004.

4. Sociedade Brasileira De Diabetes (SBD). Tratamento e acompanhamento do Diabetes Mellitus: Diretrizes da SBD. Rio de Janeiro; 2007.

5. World Health Organization. Physical status: the use and interpretation of anthropometry. Geneva: World Health Organization; 1995. (Technical Report Series, 854).

6. Higa R, Lopes MHBM, Reis MJR. Fatores de risco para incontinência urinária na mulher. Rev Esc Enferm USP. 2008; 42 (1):187-92. http://dx.doi.org/10.1590/S0080$\underline{62342008000100025}$

7. Marini G, Piculo F, Barbosa AMP, Damasceno DC, Matheus SMM, Rudge MVC. Importância do modelo animal para testar hipóteses sobre a fisiopatologia do binômio diabetes e incontinência urinária feminina. Scientia Medica (Porto Alegre) 2011; 21 (4): 191-5.

8. Abrams P, Cardoso L, Fall M, Griffiths $D$, Rosier $P$, Ulmsten $U$, et al. The standardization of terminology of lower urinary tract function: Report from the standardization sub-committee of the international continence society. Urology. 2003; 61(1):37-49. 
http://dx.doi.org/10.1016/S0090-

4295(02)02243-4

9. Guarisi T. Incontinência urinária em mulheres climatéricas: estudo epidemiológico, clínico e urodinâmico. [tese]. Campinas, SP: Faculdade de Ciências Médicas da Universidade Estadual de Campinas; 2000.

10. Simeonova Z, Milson I, Kullendorff AM, Molander $U$, Bengtsson $C$. The prevalence of urinary incontinence and its influence on the quality of life in women from urban Swedish population. Acta Obstet Gynecol Scand.1999;78(6):546-51. http://dx.doi.org/10.1080/j.1600$\underline{0412.1999 .780613 . x}$

11. O'Brien J, Austin M, Seth $P, O^{\prime}$ Boyle P. Urinay incontinence: prevalence, need for treatment, and effectiveness of intervention by nurse. BMJ. 1991;303(6813):1308-12. http://dx.doi.org/10.1136/bmj.303.6813.130 $\underline{8}$

12. Oliveira JMS, Prevalência da Incontinência Urinária e sua associação com a obesidade em mulheres na transição menopausal e após menopausa; São Paulo, 2010; Dissertação, Universidade de São Paulo Faculdade de Saúde Pública.

13. Pereira WMP. Dissertação Prevalência de depressão e ansiedade associada à obesidade em mulheres na transição e após menopausa. FSP/USP: 2007-2010.

14. Associação Brasileira para o Estudo da Obesidade e da Síndrome Metabólica (ABESO), Diretrizes Brasileira de Obesidade. São Paulo 3ạed, 2009.

15. Park HS, Cho S, Choi WH, Kim YS. The metabolic syndrome and associated lifestyle factors among South Korean adults. Int J Epidemiol. 2004; 33:328-36. http://dx.doi.org/10.1093/ije/dyh032

16. Instituto Brasileiro de Geografia e Estatística (IBGE). Pesquisa Nacional por
Amostra de Domicílio: síntese de indicadores 2004. Rio de Janeiro: IBGE, 2005a.

17. Fenner DE, Genberg B, Brahma $P$, Marek L, Delancey JO. Fecal and urinary incontinence after vaginal delivery with anal sphincter this disruption in on obstetrics unit in the Uniteds States. Am J Obstet Gynecol. $2003 ; 189(6)$ :

1543-9. http://dx.doi.org/10.1016/i.ajog.2003.09.030

18. Centro Coordenador do IPAQ no Brasil. Manual de Fisioterapia na Reabilitação Cardiovascular. UMEDA I I K. 2006; 31, 32.

19. Tamanini JTN, D'ancona CAL, Botega NJ, Netto Junior NR. Validação do “King's Health Questionnaire" para o português em mulheres com incontinência urinária. Rev Saúde Pública. 2003;2(37):203-11. http://dx.doi.org/10.1590/S0034$\underline{89102003000200007}$

20. Beuttenmuller L, Cader SA, Macena RHM, Araujo NS, Nunes EFC, Dantas EHM. Contração muscular do assoalho pélvico de mulheres com incontinência urinária de esforço submetidas a exercícios e eletroterapia: um estudo randomizado. Fisioter Pesq. 2011;18(3): 210-6.

21. Cummings $J M$, Rodning $C B$. Urinary Stress Incontinence Among Obese Women: Review of Pathophysiology Therapy. Int Urogynecol J (2000) 11:41-4. http://dx.doi.org/10.1007/s001920050008

22. Han MO, Lee NY, Park HS. Abdominal obesity is associated with stress urinary incontinence in Korean women. Int Urogynecol J. 2005; 17: 35-9. http://dx.doi.org/10.1007/s00192-005-1356$\underline{8}$

23. Castro LA, Sobottka W, Baretta G, Freitas ACT. Efeitos da cirurgia bariátrica na função do assoalho pélvico. ABCD Arq Bras Cir Dig. 2012;25(4):263-8. 
http://dx.doi.org/10.1590/S0102-

\section{0}

24. Oliveira E, Takano CC, Sartori JP, Araújo MP, Pimentel SHC, Sartori MGF et al. Trato urinário, assoalho pélvico e ciclo gravídico-puerperal. Femina. 2007;35:89-94.

25. Knorst MR, Royer CS, Basso DMS, Russo JS, Guedes RG, Resende TL. Avaliação da qualidade de vida antes e depois de tratamento fisioterapêutico para incontinência urinária. Fisioter Pesq. 2013;20(3):204-9.

http://dx.doi.org/10.1590/S1809$\underline{29502013000300002}$

26. Parazzini F, Chiaffarino F, Lavezzari M, Giambanco V, on behalf of VIVA Study Group. Risk factors for stress, urge or mixed urinary incontinence in Italy. BJOG. 2003; 110 (10): 927 - 33. http://dx.doi.org/10.1111/i.1471$\underline{0528.2003 .02343 . x}$

27. Leroy LS, Lopes MHBM. A incontinência urinária no puerpério e o impacto na qualidade de vida relacionada à saúde. Rev. Latino-Am. Enfermagem mar.abr. 2012;20(2):[08 telas].

28. Virtuoso JF, Mazo GZ, Menezes EC. Incontinência urinária e função muscular perineal em idosas praticantes e não praticantes de atividade física regular. Rev Bras Fisioter, 2011;15(4):310-7. http://dx.doi.org/10.1590/S1413-

$\underline{35552011005000014}$

Recebido para publicação em 19/08/2014

Revisado em 29/08/2014

Aceito em 10/09/2014 